\title{
RESTOS CAUDAIS DE TITANOSAURIDAE DA FORMAÇÃO ADAMANTINA (TURONIANO-SANTONIANO), SÍTIO DO PRATA, ESTADO DE MINAS GERAIS, BRASIL
}

\author{
EDIMILSON BATISTA DE ALMEIDA \\ Laboratório de Macrofósseis, UFRJ, Av. Brigadeiro Tromposki, s/n, Ilha do Fundão, 21949-900, \\ Rio de Janeiro, RJ, Brasil.edimilson.b.a@bol.com.br,
}

LEONARDO DOS SANTOS AVILLA

Setor de Herpetologia, Departamento de Vertebrados, Museu Nacional, UFRJ, Quinta da Boa Vista, s/n, 20940-040, Rio de Janeiro, RJ, Brasil. lavilla@compuland.com.br

CARLOS ROBERTO DOS ANJOS CANDEIRO

Museu de Minerais e Rochas, Instituto de Geografia, UFU, Av. João Naves de Ávilla, 2160, Sta. Mônica, 38400-600, Uberlândia, MG, Brasil. candeiro@yahoo.com.br

\begin{abstract}
RESUMO - Restos ósseos atribuídos a Titanosauridae são bastante comuns no Cretáceo do Brasil, principalmente na Bacia Bauru. O sítio estudado dista $40 \mathrm{~km}$ da cidade do Prata (MG) na rodovia Prata-Campina Verde $\left(19^{\circ} 27^{\prime} 26.0^{\prime \prime} \mathrm{S}, 49^{\circ} 14^{\prime} 42.6^{\prime \prime} \mathrm{W}\right)$. O material aqui descrito compreende uma vértebra caudal associada ao seu respectivo arco hemal. A vértebra em questão encontra-se totalmente preservada, com exceção de pequenas arestas fragmentadas. Estas fraturas permitem a visualização da composição porosa da vértebra. $\mathrm{O}$ centro vertebral é ântero-posteriormente longo e relativamente baixo, tipo procélico, e apresenta a face articular posterior em formato de coração. O canal hemal, aberto dorsalmente, sugere a atribuição deste material aos Camarasauromorpha. A vértebra em questão é atribuída a Titanosauridae por apresentar uma das sinapomorfias deste táxon: vértebras caudais procélicas. A face articular posterior cordiforme e prezigapófises alongadas permitem relacionar este material aos gêneros Gondwanatitan e Aeolosaurus. Recentemente, um Titanosauridae foi citado para a mesma localidade, sem apresentar, no entanto, as características diagnósticas supracitadas para Gondwanatitan e Aeolosaurus. Um estudo mais detalhado deverá ser feito com os materiais de Gondwanatitan faustoi para poder avaliar a validade dessa espécie.
\end{abstract}

Palavras-chave: vértebras caudais, Titanosauridae, Formação Adamantina, Cretáceo superior, Brasil.

\begin{abstract}
CAUDAL REMAINS OF THE TITANOSAURIDAE FROM ADAMANTINA FORMATION (TURONIAN-SANTONIAN), PRATA SITE, MINAS GERAIS STATE, BRAZIL. Titanosauridae remains are very common fossils in the Brazilian Cretaceous outcrops. The site were the vertebrae was found $\left(19^{\circ} 27^{\prime} 26.0^{\prime \prime} \mathrm{S}\right.$ e $49^{\circ} 14^{\prime} 42.6^{\prime \prime} \mathrm{W}$ ) stays approximately $40 \mathrm{~km}$ from Prata town (MG), on the margin of the Prata-Campina Verde road. A Titanosauridae caudal vertebrae associated to its chevron ir reported here. The vertebral centrum is deep and relatively low, procelic, and bears a cordiform anterior articular facet. A dorsally open haemal canal indicates that the caudal vertebrae belongs to a Camarasauromorpha. We have securely associated this vertebrae to Titanosauridae as it bears one of the synapomorphies of this taxon - procelic caudal vertebrae. The cordiform posterior articular facet and wide prezigapophyses allowed us to related the vertebrae in question to the genera Gondwanatitan and Aeolosaurus. Recently, a titanosaurid was reported for the same locality here studied. However, it does not present the diagnostic characters of Gondwanatitan and Aeolosaurus. A more detailed study should be made with the materials of Gondwanatitan faustoi to evaluate the validity of this species.
\end{abstract}

Key words: caudal vertebrae, Titanosauridae, Adamantina Formation, Upper Cretaceous, Brazil.

\section{INTRODUÇÃO}

Os Titanosauridae são os dinossauros saurópodes mais representativos do Gondwana, com uma abundante ocorrência em sedimentos da América do Sul (Powell, 2003). Este grupo teve uma ampla distribuição paleogeográfica durante o Cretáceo, com registros também em áreas do Laurásia (Novas, 1997).
Restos ósseos de titanossaurídeos, principalmente Aeolosaurus, são encontrados em sedimentos das formações Allen (Salgado \& Coria, 1993), Los Alamitos (Powell, 1987; Bonaparte, 1992; Salgado et al., 1997a) e Angostura Colorada (Powell, 1986) do Cretáceo Superior da Argentina. No Brasil, esses titanossaurídeos são reportados nas formações Adamantina e Marília (Cretáceo Superior), respectivamente nos esta- 
dos de São Paulo de Minas Gerais (Bertini et al., 1999a, 1999b, 2000; Santucci \& Bertini, 2001). Novos restos de titanossaurídeos foram descobertos em 2002 em uma expedição conjunta do Laboratório de Macrofósseis (Departamento de Geologia, Universidade Federal do Rio de Janeiro) e do Museu de Minerais e Rochas (Instituto de Geografia, Universidade Federal de Uberlândia), na localidade Serra da Boa Vista, Sítio do Prata, Formação Adamantina, município do Prata, Estado de Minas Gerais (Figura 1). Nessas camadas têm sido registrado dezenas de restos de titanossaurídeos (Henriques et al., 2002; Marinho, 2003; Candeiro et al., 2003), Abelisauridae (Marinho, 2003; Moraes et al., 2003), terópodes, tartarugas e crocodilianos (Goldberg et al. 1995a,b; Silva et al., 2001). Como de parte dessa assembléia fossilífera, descreve-se aqui uma vértebra caudal de Titanosauridae, de sedimentos de idade TuronianoSantoniano da Formação Adamantina do Estado de Minas Gerais. O material está depositado na Coleção de Répteis, Departamento de Geologia, Universidade Federal do Rio de Janeiro (UFRJ-DG 270-R).

\section{LOCALIZAÇÃOEGEOLOGIA}

O material fossilífero provém da localidade chamada Serra da Boa Vista, Rodovia Prata-Campina Verde (19²7'26.0”'S; $\left.49^{\circ} 14^{\prime} 42 \cdot 6^{\prime \prime} \mathrm{W}\right)$. Esta localidade fica aproximadamente a 40 km oeste da cidade de Prata, na região do Triângulo Mineiro, Minas Gerais, Brasil (Figura 1).

A Formação Adamantina foi definida por Soares et al. (1980), que reconheceram os sedimentos desta unidade nos estados de Goiás, Mato Grosso do Sul, Minas Gerais e São Paulo, e incluindo-a no Grupo Bauru, Bacia do Paraná. A partir de observações baseadas nas variações de litologia regional dessa unidade, Barcelos (1984) subdividiu a referida formação nos membros Araçatuba e São José do Rio Preto.

Os sedimentos Sítio do Prata são considerados como pertencentes ao Grupo Bauru, Bacia Bauru, proposta essa utilizada neste trabalho (Fernandes \& Coimbra, 1996). Segundo Fernandes \& Coimbra (1996) o Grupo Bauru, na região do Triângulo Mineiro, é constituído pelas formações Adamantina, Uberaba e Marília (Figura 1). Essas rochas ocorrem sobrepostas a basaltos da Formação Serra Geral (Grupo São Bento, Bacia do Paraná).

Segundo Goldberg \& Garcia (2000) os sedimentos da Formação Adamantina são constituídos por grãos de arenitos avermelhados finos a muito finos com estratificação cruzada. Nesta unidade ocorrem, ainda, conglomerados intraformacionais e subordinados a folhelhos vermelhos, os quais são massivos ou com laminação plano-paralelas. O topo dessa unidade na Serra da Boa Vista (Sítio do Prata) é caracterizada
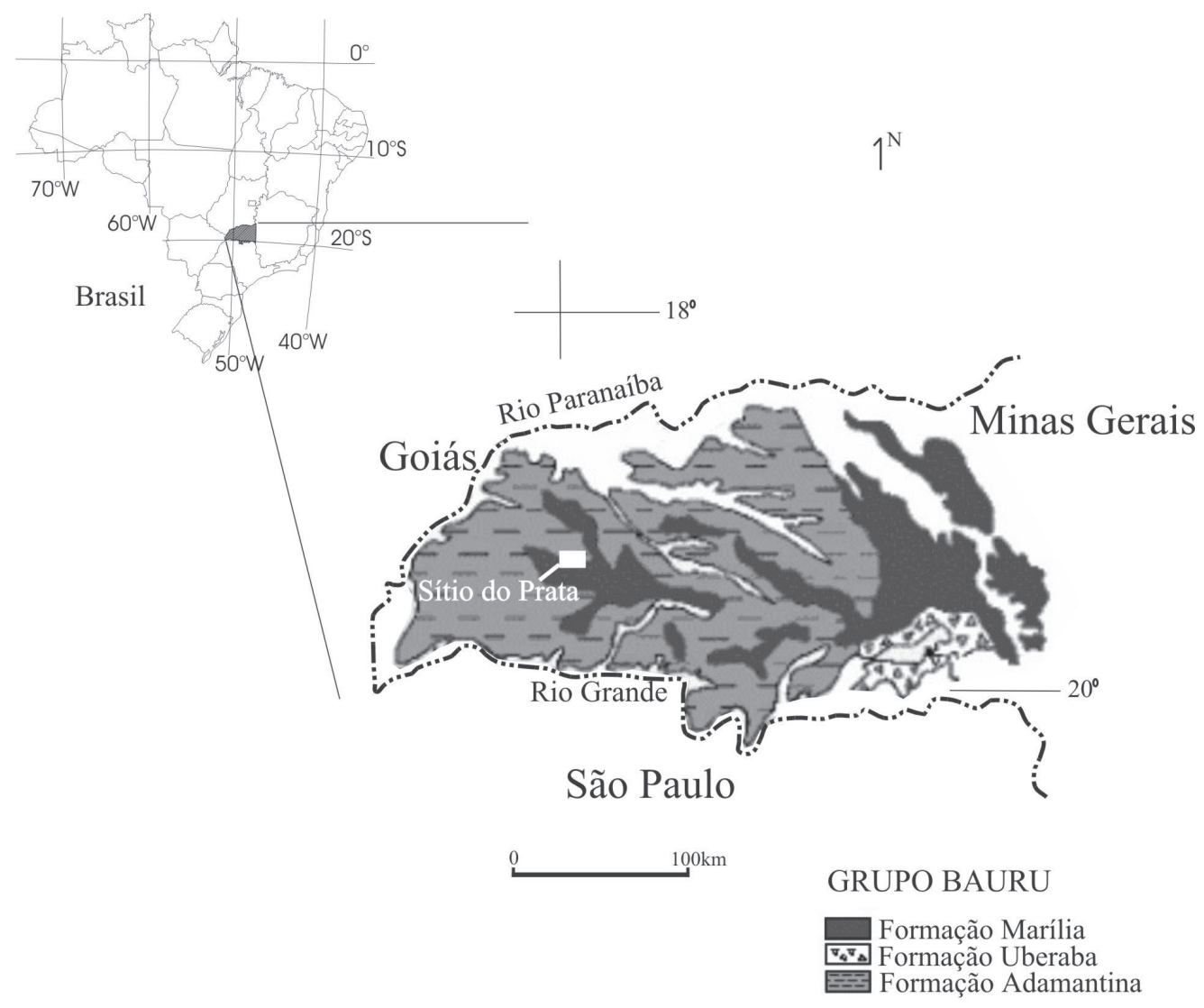

Figura 1. Mapa geológico da Bacia Bauru no Triângulo Mineiro com a localização do Sítio do Prata (modificado de Fernandes \& Coimbra, 1996).

Figure 1. Geological map of the Bauru Group in the Triângulo Mineiro region with location of the Prata Site (modified from Fernandes \& Coimbra, 1996). 


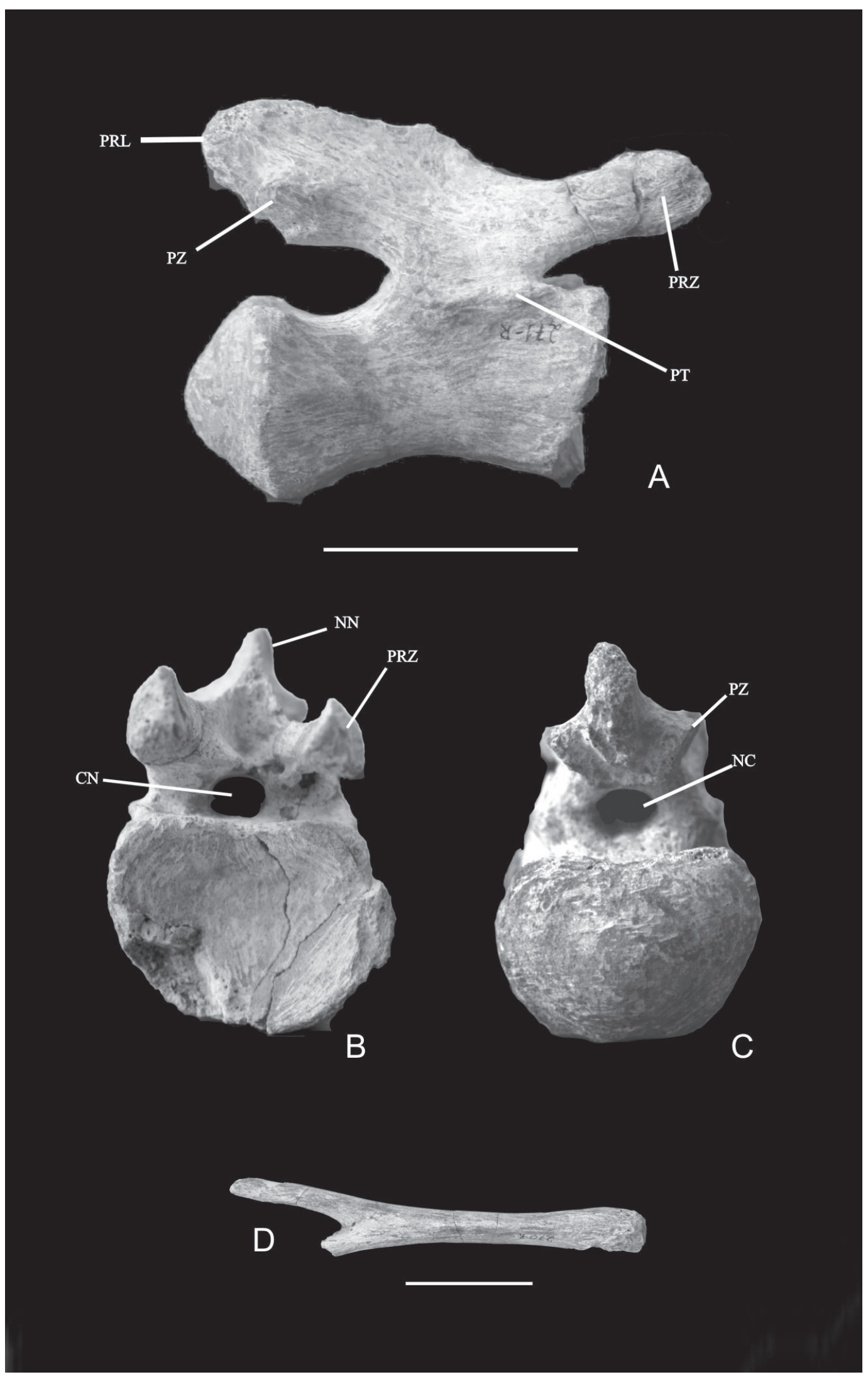

Figura 2. Vértebra caudal de Titanosauridae, UFRJ-DG 270-R. Vista lateral (A), anterior (B) e posterior (C), arco hemal (D). Abreviaturas: CN, canal neural; PRL, lâmina pré-espinhal; PRZ, pré-zigapófise; PT, processo transverso; PZ, pós-zigapófise; SN, espinho neural. Escala $=5 \mathrm{~cm}$

Figure 2. Titanosauridae caudal vertebrae UFRJ-DG 270-R. Lateral view (A), anterior (B) and posterior (C), hemal arch (D). Abbreviations: CN, neural canal; PRZ, pre-zigapophysis; PT, transverse process; PZ, postzygapophysy; SN, neural spine. Scale bar $=5 \mathrm{~cm}$. 
por conglomerados e arenitos de granulometria fina a média com estratificação cruzada, além de abundantes nódulos e horizonte de concreções carbonáticas

A Formação Adamantina tem sido datada como Turoniano-Santoniano, com base no conteúdo de ostracodes e carófitas encontrados nos sedimentos desta unidade no estado de São Paulo (Dias-Brito et al., 2001).

\section{SISTEMÁTICAPALEONTOLÓGICA}

\section{SAURISCHIA Seeley, 1887 \\ SAUROPODA Marsh, 1878 \\ TITANOSAURIA Bonaparte \& Coria, 1997 \\ TITANOSAURIDAE Lyddeker, 1893}

(Figura 2)

Material. UFRJ-DG 270-R, vértebra caudal associada a seu respectivo arco hemal.

Descrição. A vértebra em questão encontra-se muito bem preservada, com exceção de pequenas arestas fragmentadas, que não impedem a caracterização da mesma (Figura 2A). Essas fraturas permitem a visualização da composição porosa da vértebra. A superfície da vértebra possui marcas de fortes inserções musculares.

O centro vertebral é alongado ântero-posteriormente $(101,21 \mathrm{~mm})$ e relativamente baixo $(39,11 \mathrm{~mm})$ em relação ao comprimento total do espécime. A face ventral do centro é levemente côncava (Figura 2A). O centro é do tipo procélico e apresenta a face articular posterior cordiforme (Figura 2C), bastante convexa, com um profundo sulco central, provavelmente relacionado à inserção de ligamentos vertebrais. Por sua vez, a face articular anterior é bastante côncava, e uma de suas bordas ventrais encontra-se parcialmente fragmentada, evidenciando a sua porosidade (Figura $2 \mathrm{~B}$ ).

O processo transverso é bastante marcado e posicionado na sutura centro-neural, e apresentando duas protuberâncias bem demarcadas, localizadas nas porções anterior e posterior.

A base do arco neural é posicionada anteriormente em relação ao centro vertebral. O canal neural apresenta um aspecto oval, tem o diâmetro vertical menor $(13,63 \mathrm{~mm})$ que o horizontal $(18,37 \mathrm{~mm})$.

O espinho neural é relativamente longo e direcionado posteriormente. Apresenta lâmina pré-espinhal (PRL) bastante desenvolvida, sendo mais delgada na porção proximal e mais larga distalmente. As lâminas espinhais da prézigapófise encontram-se bem desenvolvidas e fundem-se na base da parte superior da pós-zigapófise. A pré-zigapófise é longa, e sua porção articular transpõem a porção anterior do centro vertebral. As facetas articulares das pré-zigapófises são de formato ovalado, onde o eixo maior é ântero-posterior, e são levemente voltadas dorsalmente. A pós-zigapófise é bastante pronunciada, e suas facetas articulares também são de formato ovalado, porém o eixo maior é dorso-ventral e são levemente voltadas ventralmente.

$\mathrm{O}$ arco hemal é reto e comprimido lateralmente, apresentando 31,69 mm de espessura na extremidade terminal e 151,35 mm de comprimento. A porção proximal (articulação com o cen- tro vertebral) está fragmentada, entretanto o arco hemal em forma de "Y" está presente (Figura 2D). O arco hemal também apresenta em sua superfície marcas de fortes inserções musculares.

\section{DISCUSSÃOECONCLUSÕES}

A presença do canal hemal aberto dorsalmente sugere que esta vértebra caudal seja de um Sauropoda Camarasauromorpha (ver Salgado et al., 1997b). Entretanto, Wilson \& Sereno (1998) indicam esta característica como sinapomorfia de um novo táxon mais abrangente, Macronaria, que, além dos Camarasauromorpha (sensu Salgado et al., 1997b), compreende Haplocanthosaurus Hatcher, 1903. Wilson (2002) apontou que quase todos os neosaurópodes possuem canal hemal aberto, sendo que a condição derivada de canal hemal fechado dorsalmente é sinapomorfia dos Diplodocoidea + Diacraeosauridae. Os Theropoda mais basais (principalmente Eoraptor) e prosaurópodes apresentam o canal hemal com uma margem dorsal côncava (Wilson \& Sereno, 1998). Essa condição também é observada em saurópodes basais (Wilson \& Sereno op. cit.), principalmente em Vulcanodon karibaensis Cooper, 1984, como também nos Diplodocidae (Gilmore, 1936). A vértebra aqui descrita compartilha um canal hemal aberto dorsalmente com camarasaurídeos (Haplocanthosaurus e Brachiosaurus brancai) e titanossaurídeos (Salgado et. al., 1997b; Wilson \& Sereno, 1998). O formato de "Y" do arco hemal sugere que este deva pertencer a um Titanosauria. Wilson \& Sereno (1998) indicam essa condição primitiva para Dinosauria, em relação ao padrão bifurcado do arco hemal, com projeções anteriores e posteriores. Entretanto, em Titanosauria, haveria uma reversão a condição primitiva, e nesse caso uma sinapomorfia do táxon.

A vértebra descrita é atribuída a Titanosauridae por apresentar uma das sinapomorfias deste táxon: vértebras caudais extremamente procélicas, apresentando faces articulares em "ball and socket" (Salgado et. al., 1997b).

Kellner \& Azevedo (1999) descreveram para a Formação Adamantina um titanossaurídeo, Gondwanatitan faustoi, apontando como uma das autapormofias do táxon novo, faces articulares distais cordiformes nas vértebras proximais e médias. A vértebra caudal, aqui descrita também apresenta tal condição (Figura 2C). Entretanto, Santucci \& Bertini (2001) sugerem que tal condição está presente também em Aeolosaurus Powell, 1987, e baseado nessa, como também nas outras autapomorfias de Gondwanatitan (Kellner \& Azevedo, 1999), propõem a sinonímia desse gênero com Aeolosaurus. Contudo, facetas articulares cordiformes também ocorrem em algumas vértebras caudais de diferentes titanossaurídeos (Pellegrinisaurus powelli e "Titanosaurus" araukanikus), como resultado da redução das superfícies de inserção do músculo caudofemoralis não devendo essa característica ser usada como autapomorfia de Gondwanatitan (Salgado \& Garcia, 2002). Ainda, as pré-zigapófises longas presentes no exemplar do Prata (Figura 2A), são apontadas por Salgado \& Coria (1993) como característica de Aeolosaurus. Sendo assim, essas características nos permitem relacionar a vértebra caudal aqui descrita aos gêneros Gondwanatitan e 
Aeolosaurus. Um estudo mais detalhado deverá ser feito com os materiais de Gondwanatitan faustoi para poder avaliar a validade desta espécie.

\section{AGRADECIMENTOS}

Agradecemos a Leonardo Salgado (Universidad Nacional del Comahue, Argentina) pela valiosa revisão e comentários feitos ao manuscrito. A Lílian P. Bergqvist (UFRJ) pela leitura do manuscrito e suporte no trabalho de campo. A Érika Abrantes (UFRJ), Adelino Carvalho, Cláudia R. Candeiro, Jorge Albuquerque, Flávio Oliveira e Patrícia Tavares (UFU) no auxílio nas escavações e a Adriano R. Santos (UFU) pela autorização do trabalho de campo na Serra da Boa Vista, Prata-MG. Essa pesquisa foi financiada pela FAPERJ (projeto E26/170.189.2000), CAPES e UFRJ. Esse trabalho é uma contribuição para o Instituto Virtual de Paleontologia/FAPERJ.

\section{REFERÊNCIAS}

Barcelos, J.H. 1984. Reconstrução paleogeográfica da sedimentação do grupo Bauru baseada na sua redefinição estratigráfica parcial em território paulista e no estudo preliminar fora do estado de São Paulo. Instituto de Geociências e Ciências Exatas, Universidade Estadual Paulista-Rio Claro, Tese de Livre Docência, 191 p.

Bertini, R.J.; Santucci, R.M. \& Arruda-Campos, A.C. 1999a. First occurrence of Aeolosaurus (Sauropoda, Titanosauridae) in Bauru Group of the Paraná Basin, Brazil. In: CONGRESSO BRASILEIRO DE PALEONTOLOGIA, 16, 1999. Boletim de Resumos, Crato, SBP, p. 27-28.

Bertini, R.J.; Santucci, R.M. \& Ribeiro, L.C. B. 1999b. O titanosáurido Aeolosaurus sp. (Saurischia, Sauropoda) no Membro Serra da Galga da Formação Marília, Grupo Bauru do Triângulo Mineiro. In: SIMPÓSIO DE GEOLOGIA DO SUDESTE, 6, 1999. Boletim de Resumos, São Pedro, UNESP, p. 78.

Bertini, R.J.; Santucci, R.M.; Ribeiro, L.C.B. \& Arruda-Campos, A.C. 2000. Aeolosaurus (Sauropoda, Titanosauridae) from Upper Cretaceous of Brazil. In: JORNADAS ARGENTINAS DE PALEONTOLOGÍA DE VERTEBRADOS, 16, 2000. Actas, San Luis, APA, p. 6.

Bonaparte, J.F. 1992. Uma nueva espécie de Tricodonta (Mammalia) de la Formacion Los Alamitos, Província de Rio Negro y comentários sobre su fauna de mamiferos. Ameghiniana, 29:99-100.

Candeiro, C.R.A.; Marinho, T.S.; Novais, G.T.; Franco, R.; Albuquerque, J.A.C. \& Oliveira, E.C. 2003. Previously unreported result from the Upper Cretaceous of the Paleontological Site of Prata, Minas Gerais State, Brazil. In: SIMPÓSIO BRASILEIRO DE PALEONTOLOGIA DE VERTEBRADOS, 3, 2003. Resumos, Rio de Janeiro, UERJ, p.20.

Dias-Brito, D.; Musacchio, J.C.; Castro, M.S.A.; Maranhão, J.M.; Suárez, J. \& Rodrigues, R. 2001. Grupo Bauru: uma unidade continental do Cretáceo do Brasil - concepções baseadas em dados micropaleontológicos, isotópicos e estratigráficos. Revue de Paléobiologie, 20:245-304.

Fernandes, L.A. \& Coimbra, A.M. 1996. A Bacia Bauru (Cretáceo Superior, Brasil). Anais da Academia Brasileira de Ciências, 68(2): 195-205.

Gilmore, C.W. 1936. Osteology of Apatosaurus with special reference to specimens in the Carnegie Museum. Memoirs of the Carnegie Museum, 11:175-300.
Goldberg, K. \& Garcia, A.J.V. 2000. Palaeogeography of the Bauru Group, a dinosaur-bearing Cretaceous unit, northeastern Paraná Basin, Brazil. Cretaceous Research, 21:241-254.

Goldberg, K.; Azevedo, S.A.K. \& Garcia, A.J. 1995a. A ocorrência de fósseis de dinossauros no Município de Prata, MG. In: CONGRESSO BRASILEIRO DE PALEONTOLOGIA, 14, 1995. Atas, Uberaba, SBP, p. 53.

Goldberg, K.; Garcia, A.J. \& Azevedo, S.A.K. 1995b. Geologia e paleoambiente dos sedimentos associados aos depósitos fossilíferos do Município de Prata, MG. In: CONGRESSO BRASILEIRO DE PALEONTOLOGIA, 14, 1995. Atas, Uberaba, SBP, p. 54.

Henriques, D.R.; Kellner, A.W.A.; Azevedo, S.A.K.; Santos, D.D. \& Craik, M.M.T. 2002. On a sauropod dinosaur (Titanosauridae) from the Adamantina Formation (Late Cretaceous). Journal of Vertebrate Paleontology, 22(3):64A.

Kellner, A.W.A. \& Azevedo, S.A.K. 1999. A new sauropod dinosaur (Titanosauria) from the Late Cretaceous of Brazil. National Science Museum Monographs, 15:111-142.

Marinho, T.S. 2003. As ocorrências fossilíferas do Cretáceo Superior nos Municípios do Prata e Monte Alegre de Minas, no Triângulo Mineiro. Instituto de Biologia, Universidade Federal de Uberlândia, Monografia de Graduação, 20 p.

Moraes, S.F.; Oliveira, E.C.; Albuquerque, J.A.C.; Novais, G.T.; Marinho, T.S. \& Candeiro, C.R.A. 2003. New discoveries of vertebrates fossils and the occurrence of a new fossil location in the Paleontological Site of Prata (Upper Cretaceous), Minas Gerais State, Brazil. In: SEMANA CIENTÍFICA DE ESTUDOS BIOLÓGICOS, 25, 2003. Resumos, Uberlândia, UFU, p. 17.

Novas, F.E. 1997. Abelisauridae. In: P.J. Currie \& K.Padian (eds.) Encyclopedia of Dinosaurs, Academic Press, p. 678689.

Powell, J.E. 1986. Revisión de los titanosáuridos de América del Sur. Universidad Nacional de Tucumán, Tese de Doutorado, $340 \mathrm{p}$.

Powell, J.E. 1987. The Late Cretaceous Fauna from Los Alamitos, Patagona, Argentina. Part. VI. The titanosaurids. Revista del Museo Argentino de Ciencias Naturales, 3(3):147-153.

Powell, J.E., 2003. Revision of South American Titanosaurid dinosaurs: paleobiological, paleobiogeographical and phylogenetic aspects. Records of the Queen Victoria, 111: $173 \mathrm{p}$.

Santucci, R.M. \& Bertini, R.J. 2001. Distribuição paleogeográfica e biocronológica dos titanossauros (Saurischia, Sauropoda) do Grupo Bauru, Cretáceo Superior do Sudeste Brasileiro. Revista Brasileira de Geociências, 31(3):307-314.

Salgado, L. \& Coria, R.A. 1993. El gênero Aeolosaurus (Sauropoda, Titanosauridae) em la Formacion Allen (CampanianoMaastrichtiano) de la Província de Rio Negro, Argentina. Ameghiniana, 30(2):119-128.

Salgado, L. \& Garcia, R. 2002. Variación morfológica en la secuencia de vértebras caudales de algunos saurópodos titanosaurios. Revista Española de Paleontología, 17(2):211216.

Salgado, L.; Coria, R.A. \& Calvo, J.O. 1997a. Presencia del gênero Aeolosaurus (Sauropoda, Titanosauridae) en La Formación Los Alamitos, Cretácico Superior de La Provincia de Río Negro, Argentina. Revista da Universidade de Guarulhos, 2:44-49.

Salgado, L.; Coria, R.A. \& Calvo, J.O. 1997b. Evolution of Titanosaurid Sauropods. I: Phylogenetic analysis based on the postcranial evidence. Ameghiniana, 34(1):3-32. 
Silva, E.P.; Brito, I.M.; Santos, A.R.; Candeiro, C.R.A. \& Ribeiro, L.C.B. 2001. Sítio Paleontológico do Prata, Triângulo Mineiro. In: CONGRESSO BRASILEIRO DE PALEONTOLOGIA, 17, 2001. Resumos, Rio Branco, SBP, p. 195.

Soares, P.C.; Landim, P.M.B.; Fulfaro, V.J. \& Neto, A.F.S. 1980. Ensaio de caracterização estratigráfica do Cretáceo no Estado de São Paulo: Grupo Bauru. Revista Brasileira de Geociências, 10:177-185.
Wilson, J.A. 2002. Sauropod dinosaur phylogeny: critique and cladistic analysis. Zoological Journal of the Linnaean Society, 136:217-276.

Wilson, J. \& Sereno, P.C. 1998. Early evolution and higher-level phylogeny of sauropod dinosaurs. Society of Vertebrate Paleontology, Memoir, 5:1-68. (Supplement to Journal of Vertebrate Paleontology 18). 